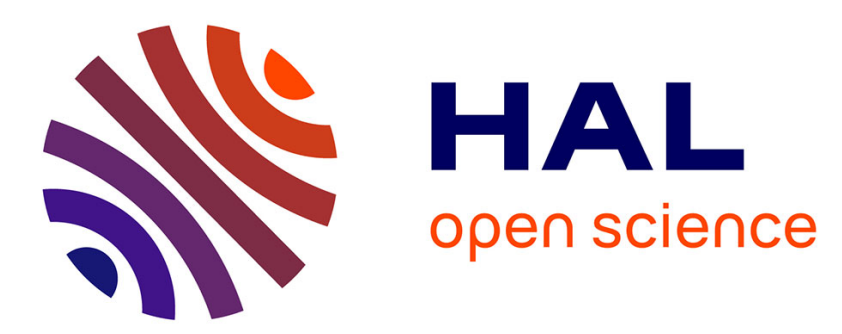

\title{
A review of the impact of hormone therapy on prefrontal structure and function at menopause \\ Y. Li, Jean-Claude Dreher
}

\section{To cite this version:}

Y. Li, Jean-Claude Dreher. A review of the impact of hormone therapy on prefrontal structure and function at menopause. Climacteric, 2021, 24 (4), pp.340-349. 10.1080/13697137.2021.1889500 . hal-03369341

\section{HAL Id: hal-03369341 https://hal.science/hal-03369341}

Submitted on 7 Oct 2021

HAL is a multi-disciplinary open access archive for the deposit and dissemination of scientific research documents, whether they are published or not. The documents may come from teaching and research institutions in France or abroad, or from public or private research centers.
L'archive ouverte pluridisciplinaire HAL, est destinée au dépôt et à la diffusion de documents scientifiques de niveau recherche, publiés ou non, émanant des établissements d'enseignement et de recherche français ou étrangers, des laboratoires publics ou privés. 


\section{Climacteric}

\section{A review of the impact of hormone therapy on prefrontal structure and function at menopause}

\section{Y. Li \& J.-C. Dreher}

To cite this article: Y. Li \& J.-C. Dreher (2021): A review of the impact of hormone therapy on prefrontal structure and function at menopause, Climacteric, DOI: 10.1080/13697137.2021.1889500

To link to this article: https://doi.org/10.1080/13697137.2021.1889500

曲 Published online: 11 Mar 2021.

Submit your article to this journal $\pi$

山 Article views: 29

Q View related articles $\widetilde{ }$

View Crossmark data \lceil 


\title{
A review of the impact of hormone therapy on prefrontal structure and function at menopause
}

\author{
Y. Li ${ }^{\mathrm{a}, \mathrm{b}}$ and J.-C. Dreher ${ }^{\mathrm{a}, \mathrm{b}, \mathrm{c}}$
}

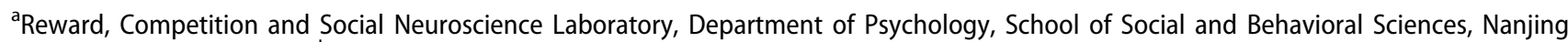

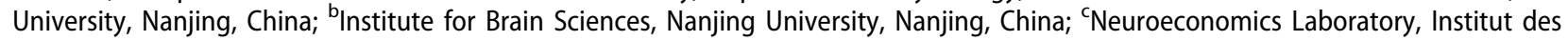
Sciences Cognitives Marc Jeannerod, CNRS UMR 5229, Bron, France

\section{ABSTRACT}

The menopause transition arises mainly from a decline in ovarian function characterized by a decrease in levels of ovarian estrogens (estradiol) and progesterone in women. Menopausal hormone therapy (MHT) has been used to counteract menopause-associated symptoms in postmenopausal women. With the development of advanced brain imaging methods, understanding MHT-related effects on brain structures and functions could help advance our understanding of the biological consequence of MHT-related effects on behavior, thereby contributing to developing new strategies for optimizing brain health during the menopause transition. This review focuses on the human research related to the impact of MHT on structural and functional organization of the prefrontal cortex in postmenopausal women. Although such MHT-related effects on brain structures and functions have only begun to be understood, it may be useful to examine present findings to identify areas for future research.

\section{ARTICLE HISTORY}

Received 17 October 2020

Revised 22 January 2021

Accepted 1 February 2021

Published online 11 March 2021

\section{KEYWORDS}

Menopausal hormone

therapy; prefrontal cortex;

hormone; estrogen;

progesterone; menopause; estrogen-only therapy; estrogen and progestogen therapy

\section{Introduction}

Due to an increase in life expectancy of women worldwide, it is estimated that globally there will be approximately 25 million women in menopause and nearly 1.2 billion postmenopausal women by the middle of this century (year 2030) [1]. The common symptoms associated with the menopause transition include both physical symptoms. such as hot flushes, night sweats, sleep disruption and vaginal dryness, and mood symptoms. such as depression, anxiety and panic attacks [2,3]. These accompanying symptoms can be distressing and sometimes seriously affect quality of life in women. It is estimated that at least $80 \%$ of postmenopausal women have experienced at least one menopausal symptom in their lifetime [4]. Although menopause is a natural phase of a woman's life cycle that occurs as a part of aging in women, it may increase risks for health problems. For example, early menopause has been found to be associated with an increased risk of cardiovascular disease [5-7]. Meanwhile, there is also increasing evidence that the menopause transition can have an impact on women's cognitive functioning $[2,8]$. In spite of the controversial nature of the available findings, accumulating evidence suggests that the menopause transition is associated with increased vulnerability to cognitive dysfunction that is consistently manifested by decreased performance on verbal fluency and verbal memory tests [9-11]. These issues may pose a major challenge for the health-care system worldwide that aims to provide effective care for women experiencing menopause symptoms and cognitive health, thus resulting in an increasing financial burden on global economies. Over the past decade, increasing empirical efforts have been devoted to understanding the causes of women's menopause transition. These studies have made it possible to identify risk factors, establish a correct diagnosis and develop a range of effective therapeutic treatments. Based on a number of notable findings over past decades, there is a consensus that menopausal changes arise mainly from a decline in ovarian function characterized by a decrease in levels of ovarian estrogens (estradiol) and progesterone in women [12]. Therefore, menopausal hormone therapy (MHT) [1] - previously known as hormone replacement therapy or hormone therapy - has been used, when appropriate, to counteract menopause-associated symptoms and, simultaneously, to evaluate its beneficial and detrimental effects on cognitive performance [13-16].

MHT usually involves treatment with any estrogenic or estrogen-like therapy alone or with combined estrogen-progestogen therapy [17]. By convention, here, we refer to these as estrogen-only therapy (ET) or estrogen replacement therapy and as estrogen-progestogen therapy (EPT), respectively. Previous research has shown the efficacy of MHT to treat menopause-associated symptoms (e.g. relief of hot flushes, vasomotor symptoms, mood swings and sleep disturbance) and improve cognitive performance (e.g. verbal memory and executive function), although this is not without controversy [18-22]. Moreover, consensus has begun to emerge that 
understanding the basic biological processes of MHT-related effects on cognitive function would greatly contribute to effective evaluation of such effects and help to develop new strategies for optimizing mental health during the menopausal transition in women. Along this line of research, there has been a surge of interest in the use of neuroimaging tools (e.g. functional magnetic resonance imaging [fMRI]) to investigate the neurobiological consequences of $\mathrm{MHT}$ during the menopause transition, although such MHT-related effects on brain structures and functions have only begun to be understood and are still being debated [23-25].

A few recent systematic reviews have attempted to synthesize available findings on MHT-related effects on cognitive brain structures and functions to highlight areas available for future work [25-27]. These endeavors have, undoubtedly, contributed to our overall understanding of such effects. However, we believe that a more precise understanding of the neurobiological effects of MHT may benefit from a critical evaluation of the literature limited to MHT-related effects on the structural and functional organization of a specific brain area: the prefrontal cortex (PFC). Here, we hope to provide a comprehensive review of MHT-related effects on the structural and functional organization of the PFC in menopausal women. We have focused on the PFC mainly because the majority of previous studies have found positive effects of MHT on PFC-dependent cognitive functions in menopausal women, such as inhibition control, mental flexibility, working memory and planning ability $[21,28-30]$. In this review, we briefly describe the structural and functional organization of the human PFC. We then discuss studies showing brain imaging results that relate to the MHT-related effects on the structural and functional organization of the PFC during cognitive processing. We conclude with suggestions/recommendations for future research.

\section{The structural and functional organization of the human prefrontal cortex: a brief introduction}

The PFC is the part of the cerebral cortex covering the frontal lobe, which reaches its maximum volume in the human brain, where it occupies $30 \%$ of the total cortical area [31]. Over past decades, considerable efforts have been devoted to identifying the anatomy, cytoarchitecture and connectivity of this brain region using different approaches $[32,33]$. A wealth of anatomical studies of the PFC have identified multiple subdivisions at the functional, cytoarchitectonic and connectivity levels [34]. According to the widely used Brodmann's cortical scheme (cytoarchitectonic map), the PFC traditionally comprises Brodmann areas (BAs) 8-14 and BAs 44-47 (Figure 1) [31]. A number of neuroimaging studies focus on functional localizations and divisions of the human PFC. There are variations in the subdivisions of the human PFC, but the dorsolateral, dorsomedial, ventromedial
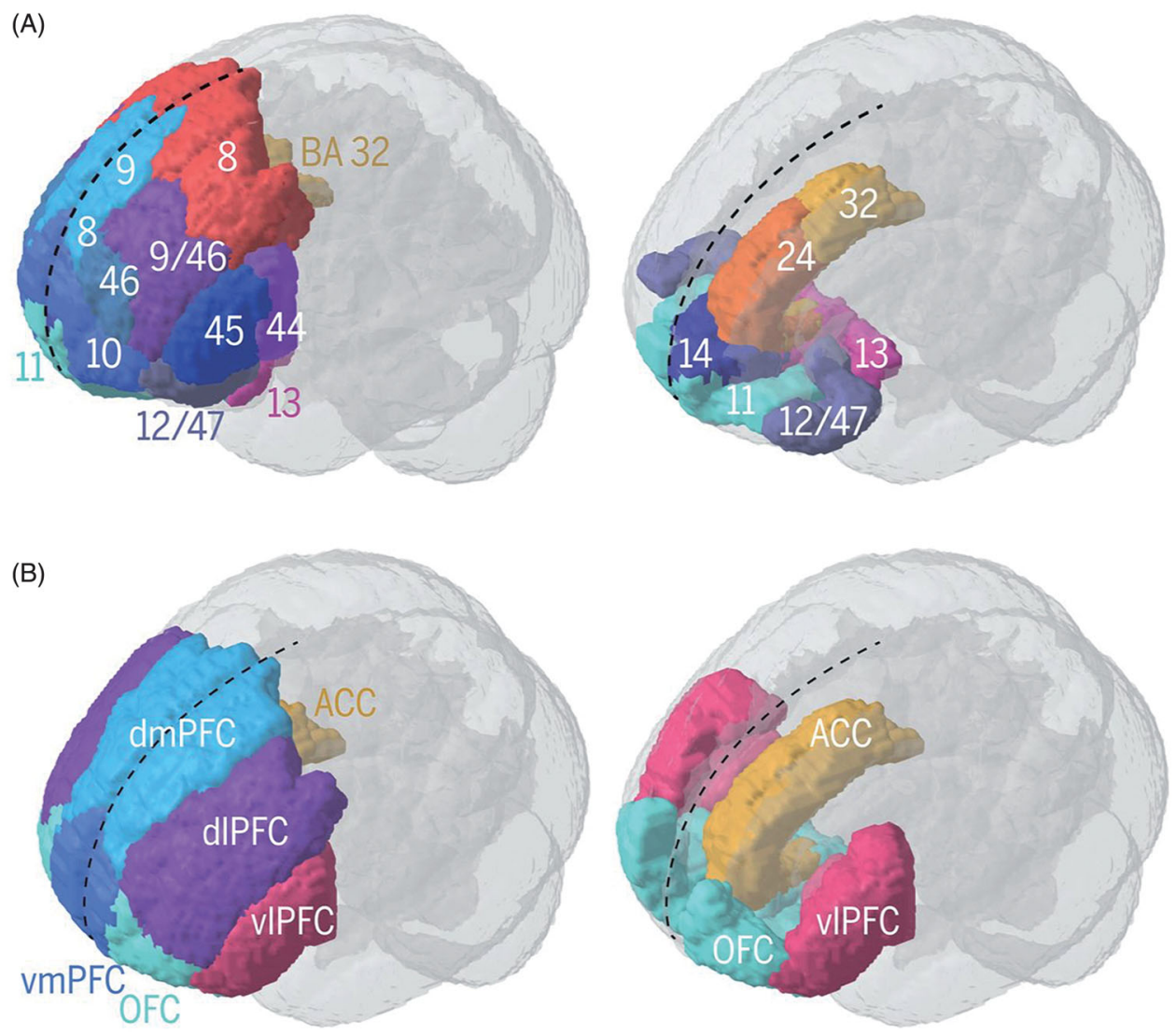

Figure 1. Anatomical and functional divisions of the human prefrontal cortex. (A) Schematic illustration of Brodmann areas (BAs) of the human prefrontal cortex (PFC). Note that not all prefrontal BAs are visible on the left panel (see right panel for medial and ventral BAs in the PFC, including the anterior cingulate cortex $[A C C]$ ). (B) Schematic illustration of common functional divisions of the human PFC, including the ACC. Dashed black line indicates sagittal midline. dIPFC, dorsolateral prefrontal cortex; dmPFC, dorsomedial prefrontal cortex; OFC, orbitofrontal cortex; vIPFC, ventrolateral prefrontal cortex; vmPFC, ventromedial prefrontal cortex. Note that this figure is from Marie Carlén Science (2017), slightly modified, with permission. 
and orbital PFC are the most common functional divisions (Figure 1) [31]. The human dorsolateral PFC is often attributed to the lateral part of BAs 8,9 and 46 , and the human dorsomedial PFC includes portions of BAs 8, 9, 10, 24 and 32. In contrast, the ventromedial PFC and the orbitofrontal cortex (OFC) are usually attributed to the anatomical structures of BAs 47, 45 and 44 and BAs 10, 11 and 47, respectively.

\section{Structural changes in the prefrontal cortex in association with hormone therapy}

We have identified nine studies involved in examining the influence of MHT on structural changes in the PFC after menopause (Table 1). An early study, using a voxel-based morphometric technique, compared the brain volume of postmenopausal women receiving ET and EPT to that of those who were receiving no hormonal treatment [35]. Postmenopausal women receiving ET and EPT showed increased gray matter volumes in the superior frontal gyrus (SFG), middle frontal gyrus (MFG), inferior frontal gyrus (IFG), anterior cingulate cortex (ACC) and medial frontal gyrus, although these differences were not observed when comparing current and past users or different types of therapy. Moreover, this study revealed a robust effect of age and ET and EPT duration on the changes in regional PFC volumes, with greater regional-specific changes in gray matter volumes in the PFC associated with increasing age and longer durations of ET and EPT. The observation of the sparing of the PFC gray matter in postmenopausal women receiving ET and EPT is further confirmed by a follow-up study comparing the brain volume of postmenopausal women receiving shortterm, middle-term and long-term ET and EPT to that of those who were receiving no hormonal treatment [43]. In this brain imaging study, the effects of duration of ET and EPT on gray matter volume in the IFG and ACC were replicated. More importantly, postmenopausal women receiving short-term and middle-term ET and EPT (less than and up to 10 years in duration) were associated with greater sparing of regionalspecific changes in PFC gray matter volume and showed better performance on measures of executive function, whereas those who were receiving long-term ET and EPT (beyond 10 years in duration) increased the degree of prefrontal deterioration. This suggests that the neuroprotective role of MHT in the PFC after menopause appears to be dependent on MHT duration, consistent with the classical critical period hypothesis [36].

Additionally, such sparing of the regional PFC volume associated with MHT was corroborated by a recent study showing increased gray matter volume in the SFG in postmenopausal women receiving ET compared with those who were receiving no hormonal treatment [37]. While these studies failed to find any structural changes in the PFC between current and past MHT users, other studies help with clarification of this issue. Boccardi et al. not only found increased gray matter in the IFG, medial frontal gyrus, OFC and ACC in both current and past ET users compared to never users, but also observed increased gray matter in the
IFG in past ET users compared to current ET users [38]. Although all of these cited brain imaging studies indicate the sparing of regional-specific structural changes in the PFC, inconsistent findings exist. Specifically, two early brain imaging studies observed a reduction of frontal lobe volume in postmenopausal women receiving ET or EPT compared with those who were receiving placebo $[39,40]$. This decline in the PFC volume has been further characterized by recent brain imaging studies showing that postmenopausal women receiving ET and EPT were associated with a decrease in regional-specific changes in the PFC gray matter volume, including the ACC, medial SFG, middle cingulate cortex, OFC, SFG, MFG and IFG, when compared with those who were receiving placebo [41] or premenopausal women [42].

Taken together, these findings are quite inconsistent, reporting both beneficial and detrimental effects of MHT on structural changes in the PFC. This discrepant pattern of results may be due to the type of MHT used. A recent study provides preliminary evidence in support of the first hypothesis, showing that postmenopausal women receiving transdermal ethinylestradiol were associated with a slower rate of SFG and MFG volume decline in the PFC than those who were receiving conjugated equine estrogens [44], indicating that the neuroprotective role of MHT in the PFC may depend on the type of ET in postmenopausal women. Meanwhile, given the very limited research thus far on the role of different synthetic progestins and progesterone on the structural organizations of the PFC in women at menopause, it may be useful for future research to address this issue.

\section{The effects of hormone therapy on the functional organization of the prefrontal cortex}

More efforts have been devoted to understanding the effects of MHT on the postmenopausal brain using cognitive paradigms over the past decade. To date, there are around 15 brain imaging studies examining the influence of MHT on the PFC function during a variety of cognitive tasks (Table 2).

\section{Memory-related function}

The great majority of brain imaging studies presented in Table 2 focused on examining the impact of MHT on prefrontal memory functions in postmenopausal women. An early fMRI study used a randomized, double-blind, placebocontrolled cross-over design to investigate how ET affects brain activity in postmenopausal women during verbal and non-verbal working memory tasks [45]. This study found that, although no performance difference was found between the ET group and the placebo group, postmenopausal women showed increased activity in the SFG and MFG during the verbal storage component of verbal working memory and increased activity in the SFG, MFG and IFG during the retrieval component of verbal working memory in the ET group compared to the placebo group. Although a later fMRI study failed to produce the effects of MHT on the prefrontal memory function between pre and post ET and EPT, possibly because of a small sample size [46], the earlier 


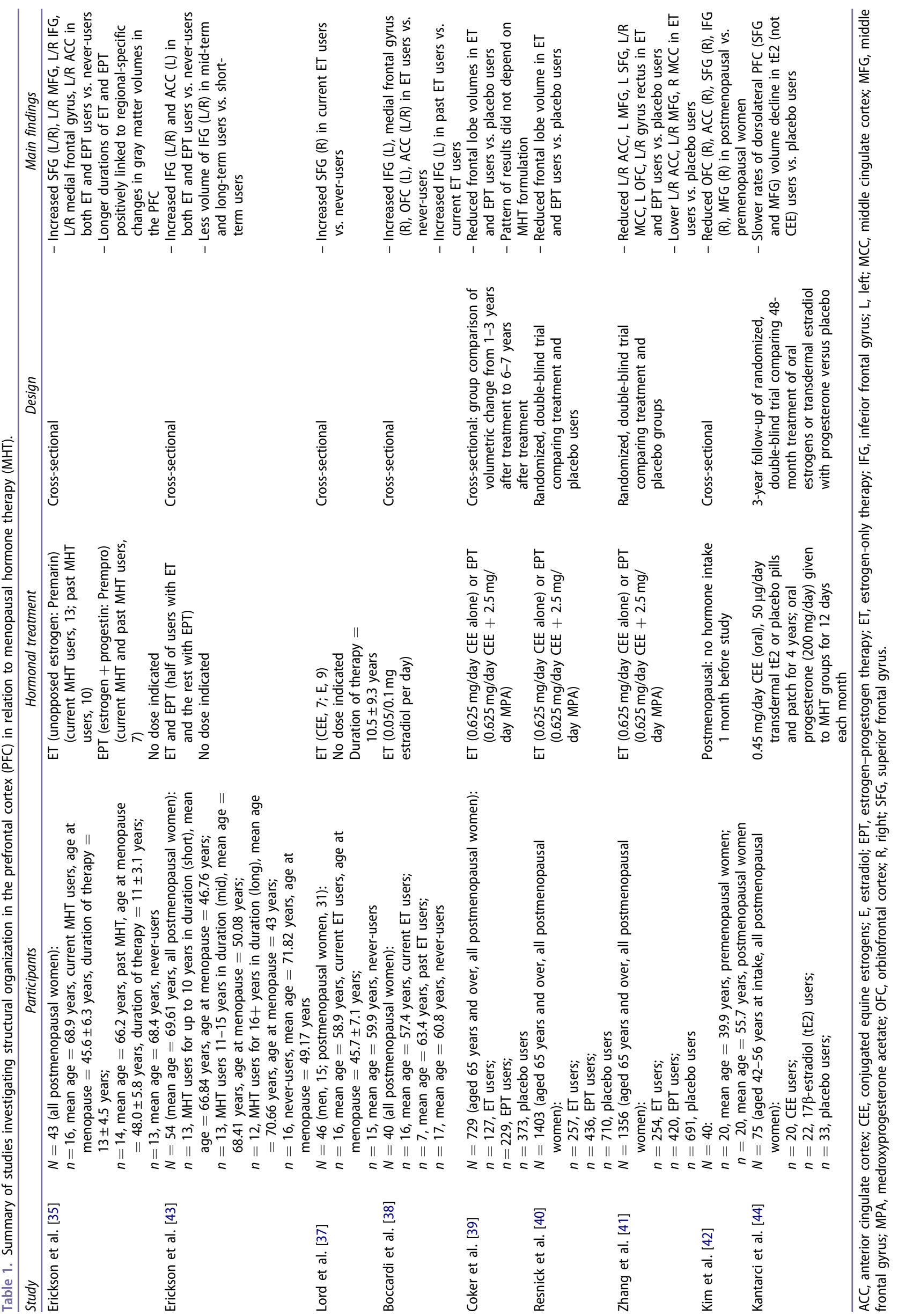




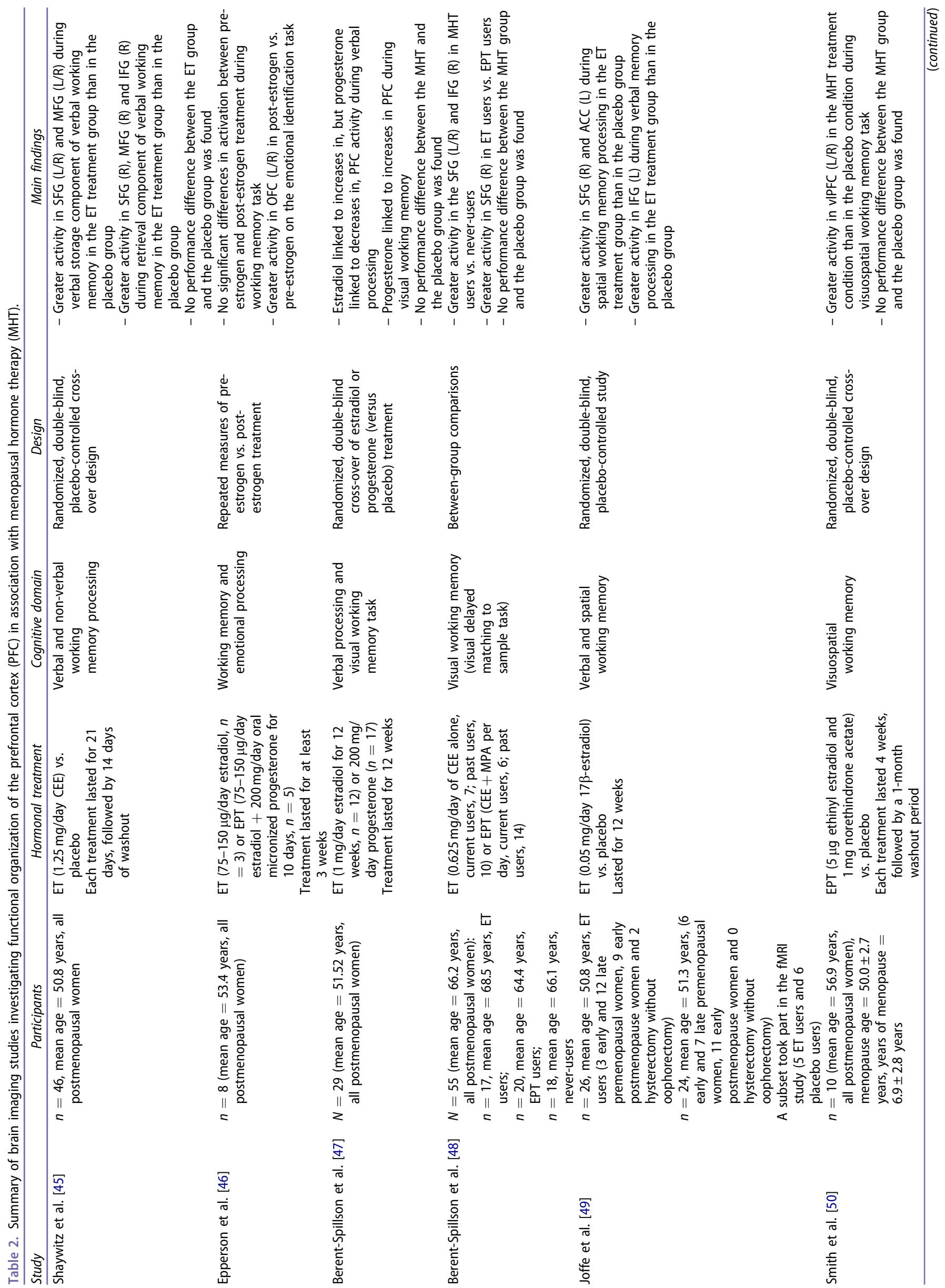



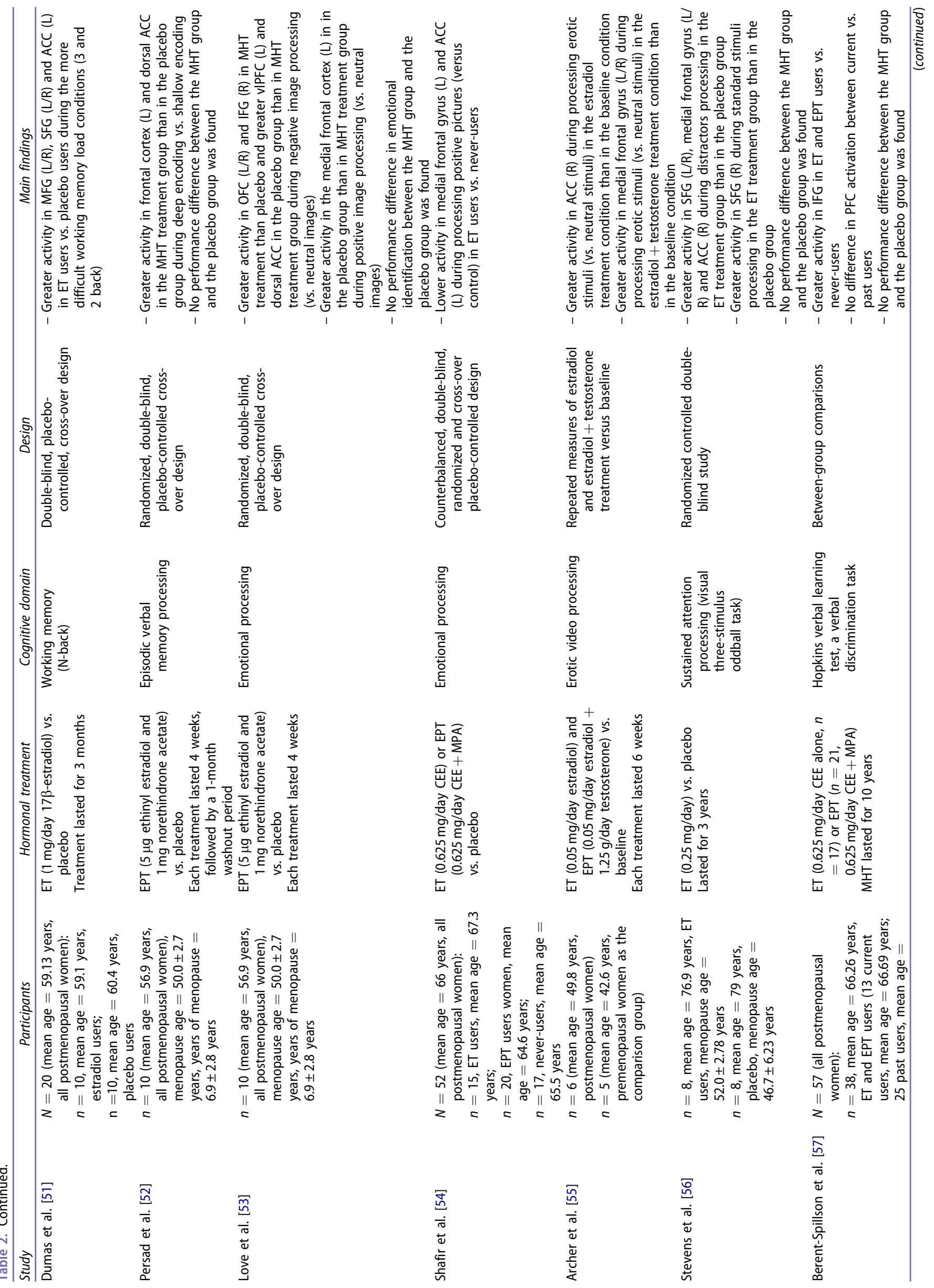
finding was corroborated by more recent fMRI studies using similar versions of the verbal working memory task. These showed increased PFC activity when postmenopausal women received ET compared with women receiving placebo [47], and increased activity in the SFG and IFG when compared to a control group [48]. The effects of MHT on prefrontal memory functions have further been observed using other types of working memory paradigms. For example, two fMRI studies with a randomized, double-blind, placebo-controlled cross-over design found increased activity in the SFG and ACC during a spatial working memory task in the ET group compared with the placebo group [49] and increased activity in the ventrolateral PFC during a visuospatial working memory task in the EPT group compared to the placebo group [50], respectively. In addition, a recent fMRI study with a randomized, double-blind, placebo-controlled cross-over design added to such a growing body of literature on the role of MHT on the prefrontal memory function by showing that postmenopausal women receiving ET showed increased activity in the MFG, SFG and ACC during more difficult working memory load conditions compared with those who were receiving a placebo [51]. Finally, in addition to the modulatory role of MHT on working memory-related PFC function, another fMRI study revealed increased activity in the ACC when postmenopausal women received EPT compared with women who had received placebo [52].

\section{Emotion-related function}

In addition to the brain imaging studies on the prefrontal memory function in association with MHT in postmenopausal women already described, another line of research attempted to examine how MHT modulates emotion-related function in the PFC in those women. To date, we found that three fMRI studies have addressed this issue. An early fMRI study with a randomized, double-blind, placebo-controlled cross-over design found increased activity in the OFC and IFG during negative image processing (vs. neutral images) when postmenopausal women received EPT compared with when they received placebo. However, no difference in the PFC activity was found during positive image processing (vs. neutral images) between the EPT condition and the placebo group [53]. This preliminary finding was further confirmed by a more recent $\mathrm{fMRI}$ study with a repeated measure of preestrogen versus post-estrogen (ET and EPT) treatment design, which showed increased activity in the OFC with estrogen treatment compared to pretreatment on the emotional identification task [46]. However, a recent study provided challenging evidence that postmenopausal women receiving ET showed reduced activity in the medial frontal gyrus and ACC compared with those who were receiving no hormonal treatment [54].

\section{Other prefrontal functions}

Given the very limited number of brain imaging studies investigating the effects of hormone replacement therapy on other aspects of PFC function in postmenopausal women, 
we have grouped these studies together in this section. Among them, an fMRI study with repeated measures of ET and ET plus testosterone treatment versus baseline design attempted to reveal how MHT impacts sexual-related function during erotic stimuli processing in the PFC [55]. Postmenopausal women receiving ET exhibited increased activity in the ACC during processing erotic stimuli (vs. neutral stimuli) compared with when they were at baseline. Meanwhile, increased activity in the medial frontal gyrus during processing erotic stimuli (vs. neutral stimuli) was observed when postmenopausal women received ET with testosterone compared with the baseline condition. In parallel, attentional and executive functions in the PFC in association with MHT have also been evaluated in postmenopausal women. An early randomized, controlled, double-blind fMRI study used a visual three-stimulus oddball task. It revealed increased activity in the SFG, medial frontal gyrus and ACC during distractors processing in postmenopausal women receiving $\mathrm{ET}$ compared with those who were receiving a placebo. In contrast, increased activity in the SFG was observed in postmenopausal women receiving ET compared with those who were receiving a placebo during standard stimuli processing [56]. This finding was further supported by a recent $\mathrm{fMRI}$ study showing increased activity in the IFG in postmenopausal women receiving either ET or EPT compared with those who were receiving a placebo [57]. Concerning cognitive control, only one fMRI study from this group using a task switching paradigm in combination with a repeated measure of EPT versus placebo design provided preliminary evidence of increased activity in the MFC, IFG, ACC and middle cingulate cortex when postmenopausal women received EPT compared with when they received a placebo [58]. Finally, given the central role of the PFC in motivation, we examined how MHT influences reward-related function in postmenopausal women. The present authors found that, in a counterbalanced, double-blind, randomized and placebocontrolled fMRI study, using a monetary reward task, there was increased activity in the anterior medial PFC at the time of rewarded outcome when postmenopausal women received EPT compared with women receiving a placebo [59].

\section{Conclusions}

Based on the findings described, it is obvious that remarkable progress has been made in understanding MHT influences on the structural and functional organization of the PFC over the past decade. Collectively, the majority of neuroimaging studies on MHT-related effects on the structural organization of the PFC indicate neuroprotective effects of MHT on regional PFC structure in postmenopausal women. Moreover, neuroimaging studies on MHT-related effects on the functional organization of the PFC mainly indicate increased brain activation patterns in different PFC subdivisions by estrogen-only or estrogen-progestogen treatment in therapeutic doses in postmenopausal women. This corroborates preclinical findings of effects of estrogen on neural structure and functions in mature animals. Although such
MHT-related effects on the PFC structures and functions have only begun to be understood and are still under debate, the available findings undoubtedly call for further research with well-powered, randomized, controlled multi-modal neuroimaging designs, especially concerning brain structural features (e.g. cortical thickness) and other aspects of PFC function (such as multi-tasking and inhibitory control) in association with the use of MHT in postmenopausal women.

\section{Acknowledgements}

The authors would like to thank Ms Xi Chen for her assistance with the literature search. Writing up this review happened during Yansong Li's newborn baby's first and second months of life. The arrival of his new family member marks the beginning of a new exciting, sweet and beautiful journey for him and his family, which represents life, hope and the promise of love, although bringing a newborn home is a stressful and life-changing event. Here, Yansong Li would like to take this opportunity to send special thanks for all the joy and smiles that his lovely boy (Hongshen Li) brings to him and his family. Wishing Shenshen a lifetime of love, health and happiness.

Potential conflict of interest The authors declare no competing interests.

Source of funding This work was supported by the Society for Social Psychology of Jiangsu Province [Grant Number 20SSXGH006]; IDEXLYON, Université de Lyon (project INDEPTH) within Programme Investissements d'Avenir [ANR-16-IDEX-0005]; LABEX CORTEX, Université de Lyon [ANR-11-LABX-0042], within Program Investissements d'Avenir [ANR-11-IDEX-007] operated by the French National Research Agency; Fondation pour la recherche Médicale [FRM DPA20140629796].

\section{References}

[1] World Health Organization. Research on the menopause in the 1990s: report of a WHO scientific group. Geneva (Switzerland): World Health Organization; 1996.

[2] Monteleone P, Mascagni G, Giannini A, et al. Symptoms of menopause - global prevalence, physiology and implications. Nat Rev Endocrinol. 2018;14:199-215.

[3] Sussman M, Trocio J, Best C, et al. Prevalence of menopausal symptoms among mid-life women: findings from electronic medical records. BMC Women's Health. 2015;15:1-5.

[4] Woods NF, Mitchell ES. Symptoms during the perimenopause: prevalence, severity, trajectory, and significance in women's lives. Am J Med. 2005;118:14-24.

[5] Rosano G, Vitale C, Marazzi G, et al. Menopause and cardiovascular disease: the evidence. Climacteric. 2007;10:19-24.

[6] Atsma F, Bartelink M-LE, Grobbee DE, et al. Postmenopausal status and early menopause as independent risk factors for cardiovascular disease: a meta-analysis. Menopause. 2006;13:265-279.

[7] Muka T, Oliver-Williams C, Colpani V, et al. Association of vasomotor and other menopausal symptoms with risk of cardiovascular disease: a systematic review and meta-analysis. PloS One. 2016;11:e0157417.

[8] Greendale GA, Karlamangla AS, Maki PM. The menopause transition and cognition. JAMA. 2020;323:1495-1496.

[9] Pertesi S, Coughlan G, Puthusseryppady V, et al. Menopause, cognition and dementia - a review. Post Reprod Health. 2019;25: 200-206.

[10] Weber MT, Maki PM, McDermott MP. Cognition and mood in perimenopause: a systematic review and meta-analysis. J Steroid Biochem Mol Biol. 2014;142:90-98.

[11] Maki PM, Henderson VW. Cognition and the menopause transition. Menopause. 2016;23:803-805. 
[12] O'Neill S, Eden J. The pathophysiology of menopausal symptoms. Obstet Gynaecol Reprod Med. 2012;22:63-69.

[13] de Villiers TJ, Hall JE, Pinkerton JV, et al. Revised global consensus statement on menopausal hormone therapy. Maturitas. 2016;91: 153-155.

[14] De Villiers T, Gass M, Haines C, et al. Global consensus statement on menopausal hormone therapy. Climacteric. 2013;16: 203-204.

[15] Hogervorst E. Estrogen and the brain: does estrogen treatment improve cognitive function? Menopause Int. 2013;19:6-19.

[16] Gava G, Orsili I, Alvisi S, et al. Cognition, mood and sleep in menopausal transition: the role of menopause hormone therapy. Medicina. 2019;55:668.

[17] Fait T. Menopause hormone therapy: latest developments and clinical practice. Drugs Context. 2019;8:212551.

[18] Pinkerton JV, Stovall DW. Reproductive aging, menopause, and health outcomes. Ann N Y Acad Sci. 2010;1204:169-178.

[19] Pinkerton JV, Stovall DW, Kightlinger RS. Advances in the treatment of menopausal symptoms. Womens Health (Lond). 2009;5: 361-384.

[20] Yoon B-K, Chin J, Kim J-W, et al. Menopausal hormone therapy and mild cognitive impairment: a randomized, placebo-controlled trial. Menopause. 2018;25:870-876.

[21] Duka T, Tasker R, McGowan J. The effects of 3-week estrogen hormone replacement on cognition in elderly healthy females. Psychopharmacology (Berl). 2000;149:129-39.

[22] Koebele SV, Mennenga SE, Hiroi R, et al. Cognitive changes across the menopause transition: a longitudinal evaluation of the impact of age and ovarian status on spatial memory. Horm Behav. 2017; 87:96-114.

[23] Mueller S, Grissom E, Dohanich G. Assessing gonadal hormone contributions to affective psychopathologies across humans and animal models. Psychoneuroendocrinology. 2014;46: 114-128.

[24] Henderson VW, Greicius MD. Functional magnetic resonance imaging and estrogen effects on the brain: cautious interpretation of a BOLD finding. Menopause. 2010;17:669-671.

[25] Bayer U, Hausmann M. Sex hormone therapy and functional brain plasticity in postmenopausal women. Neuroscience. 2011;191: 118-128.

[26] Beltz AM, Moser JS. Ovarian hormones: a long overlooked but critical contributor to cognitive brain structures and function. Ann N Y Acad Sci. 2020;1464:156-180.

[27] Comasco E, Frokjaer VG. Sundström-Poromaa I. Functional and molecular neuroimaging of menopause and hormone replacement therapy. Frontiers Neurosci. 2014;8:388

[28] File $S E$, Hartley $D E$, Elsabagh $S$, et al. Cognitive improvement after 6 weeks of soy supplements in postmenopausal women is limited to frontal lobe function. Menopause. 2005;12:193-201.

[29] Hogervorst E, Williams J, Budge $M$, et al. The nature of the effect of female gonadal hormone replacement therapy on cognitive function in post-menopausal women: a meta-analysis. Neuroscience. 2000;101:485-512.

[30] Krug R, Born J, Rasch B. A 3-day estrogen treatment improves prefrontal cortex-dependent cognitive function in postmenopausal women. Psychoneuroendocrinology. 2006;31:965-975.

[31] Carlén M. What constitutes the prefrontal cortex? Science. 2017; 358:478-482.

[32] Wilson CR, Gaffan D, Browning PG, et al. Functional localization within the prefrontal cortex: Missing the forest for the trees? Trends Neurosci. 2010;33:533-540.

[33] Brase GL, Raffone A. The key role of prefrontal cortex structure and function. Behav Brain Sci. 2006;29:22.

[34] Petrides $\mathrm{M}$, Tomaiuolo F, Yeterian EH, et al. The prefrontal cortex: comparative architectonic organization in the human and the macaque monkey brains. Cortex. 2012;48:46-57.
[35] Erickson Kl, Colcombe SJ, Raz N, et al. Selective sparing of brain tissue in postmenopausal women receiving hormone replacement therapy. Neurobiol Aging. 2005;26:1205-1213.

[36] Maki PM. The critical window hypothesis of hormone therapy and cognition: a scientific update on clinical studies. Menopause. 2013;20:695-709.

[37] Lord C, Engert V, Lupien SJ, et al. Effect of sex and estrogen therapy on the aging brain: a voxel-based morphometry study. Menopause. 2010;17:846-851.

[38] Boccardi M, Ghidoni R, Govoni S, et al. Effects of hormone therapy on brain morphology of healthy postmenopausal women: a Voxel-based morphometry study. Menopause. 2006;13: 584-591.

[39] Coker LH, Espeland MA, Hogan PE, et al. Change in brain and lesion volumes after CEE therapies: the WHIMS-MRI studies. Neurology. 2014;82:427-434.

[40] Resnick SM, Espeland MA, Jaramillo SA, et al. Postmenopausal hormone therapy and regional brain volumes: the WHIMS-MRI Study. Neurology. 2009;72:135-142.

[41] Zhang T, Casanova R, Resnick SM, et al. Effects of hormone therapy on brain volumes changes of postmenopausal women revealed by optimally-discriminative voxel-based morphometry. PloS One. 2016;11:e0150834-e0150834.

[42] Kim GW, Park K, Jeong GW. Effects of sex hormones and age on brain volume in post-menopausal women. J Sex Med. 2018;15: 662-670.

[43] Erickson Kl, Colcombe SJ, Elavsky S, et al. Interactive effects of fitness and hormone treatment on brain health in postmenopausal women. Neurobiol Aging. 2007;28:179-185.

[44] Kantarci K, Tosakulwong N, Lesnick TG, et al. Brain structure and cognition 3 years after the end of an early menopausal hormone therapy trial. Neurology. 2018;90:e1404-e1412.

[45] Shaywitz SE, Shaywitz BA, Pugh KR, et al. Effect of estrogen on brain activation patterns in postmenopausal women during working memory tasks. JAMA. 1999;281:1197-1202.

[46] Epperson CN, Amin Z, Ruparel K, et al. Interactive effects of estrogen and serotonin on brain activation during working memory and affective processing in menopausal women. Psychoneuroendocrinology. 2012;37:372-382.

[47] Berent-Spillson A, Briceno E, Pinsky A, et al. Distinct cognitive effects of estrogen and progesterone in menopausal women. Psychoneuroendocrinology. 2015;59:25-36.

[48] Berent-Spillson A, Persad CC, Love T, et al. Early menopausal hormone use influences brain regions used for visual working memory. Menopause. 2010;17:692.

[49] Joffe H, Hall JE, Gruber S, et al. Estrogen therapy selectively enhances prefrontal cognitive processes: a randomized, doubleblind, placebo-controlled study with functional magnetic resonance imaging in perimenopausal and recently postmenopausal women. Menopause. 2006;13:411-422.

[50] Smith YR, Love T, Persad CC, et al. Impact of combined estradiol and norethindrone therapy on visuospatial working memory assessed by functional magnetic resonance imaging. J Clin Endocrinol Metab. 2006;91:4476-4481.

[51] Dumas JA, Kutz AM, Naylor MR, et al. Estradiol treatment altered anticholinergic-related brain activation during working memory in postmenopausal women. Neuroimage. 2012;60: 1394-1403.

[52] Persad CC, Zubieta J-K, Love T, et al. Enhanced neuroactivation during verbal memory processing in postmenopausal women receiving short-term hormone therapy. Fertil Steril. 2009;92: 197-204.

[53] Love T, Smith YR, Persad CC, et al. Short-term hormone treatment modulates emotion response circuitry in postmenopausal women. Fertil Steril. 2010;93:1929-1937.

[54] Shafir T, Love T, Berent-Spillson A, et al. Postmenopausal hormone use impact on emotion processing circuitry. Behav Brain Res. 2012;226:147-153. 
[55] Archer JS, Love-Geffen TE, Herbst-Damm KL, et al. Effect of estradiol versus estradiol and testosterone on brain-activation patterns in postmenopausal women. Menopause. 2006;13:528-537.

[56] Stevens MC, Clark VP, Prestwood KM. Low-dose estradiol alters brain activity. Psychiatry Res. 2005;139:199-217.

[57] Berent-Spillson A, Kelley AS, Persad CC, et al. Postmenopausal hormone treatment alters neural pathways but does not improve verbal cognitive function. Menopause. 2018;25:1424.
[58] Girard R, Météreau E, Thomas J, et al. Hormone therapy at early post-menopause increases cognitive control-related prefrontal activity. Sci Reports. 2017;7:11.

[59] Thomas J, Météreau E, Déchaud $H$, et al. Hormonal treatment increases the response of the reward system at the menopause transition: a counterbalanced randomized placebocontrolled fMRI study. Psychoneuroendocrinology. 2014;50: 167-180. 